\title{
The regular performance of physical activity and the social involvement of elderly persons
}

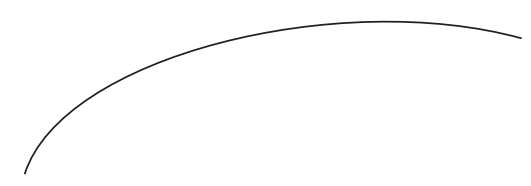

Population aging is the most visible sign of the overall improvement in quality of life and is a phenomenon that should be both celebrated and accompanied by interventions that ensure the integration of the elderly into society. Initiatives that seek to preserve the quality, meaning and right to life of individuals at advanced ages are needed to ensure that the increase in life expectancy concomitantly results in efforts to fill these extra years with a sense of selffulfillment and purpose. Considering population aging as an opportunity to be grasped rather than a problem to be solved, there is a degree of consensus that policies and initiatives focused on the material and spiritual development of this age group should be encouraged.

Based on this premise, in the 1980s the United Nations introduced the International Plan of Action On Aging, a broad set of principles and strategies that emphasize the need to promote successful aging. The recommendations contained in the plan were subsequently confirmed with the publication of the UN Principles for Older Persons. The 18 principles proposed are grouped around five key guidelines for the status of the elderly in society: a) independence; b) participation; c) care; d) self-realization; e) dignity. They aim to improve old age as the result of a continuous process, or in other words, by viewing it as a continuation of life rather than an end, and emphasizing the social dimension of aging.

The UN International Plan made it clear that one of the main methods of achieving these aims is by allowing older persons to maintain their ability to participate in society, increasing their potential to satisfy their personal ambitions. Concepts such as 'hope for an active lifestyle' or 'successful aging' illustrate the need to allocate elderly people an important role in the heart of their communities. The integration of the elderly person is, in turn, closely linked to their ability to accomplish their objectives and personal aspirations. Considering the multidisciplinary nature of these issues, it is no surprise that they have attracted attention in several areas of knowledge.

One proposed strategy that has undergone rapid growth is the study of the alterations to physical and cognitive performance capacity that come with age, as these are viewed as important components of maintaining autonomy. In addition, there are an increasing number of investigations on how such skills can be preserved by adopting active lifestyles in general, and the practice of physical exercise in particular. There is considerable evidence that a physically active life can contribute to an improvement in physical, mental and social functioning during aging.

It is believed that the major challenge with regard to the future of programs aimed at promoting the health of the elderly population is not to continue to increase life expectancy, but to improve quality of life. This assumes the preservation of the ability to perform daily activities over the years; and therefore maintain the social involvement of the elderly, something to which physical activity can obviously contribute. 
Despite the widespread acceptance of this opinion, however, it must be recognized that the access of elderly persons to physical activities remains limited. Simple consensus on the beneficial effects of physical activity is not enough to increase the participation of older people in such activities, and the prevalence of physical inactivity among individuals over 60 years of age is both evident and disturbing. Investment to identify, analyze and tackle the obstacles that prevent the involvement of the elderly in physical activity is therefore required. A greater understanding of these issues will enable the development of effective public policies aimed at continuing education, social support, information and marketing strategies and, of course, increase opportunities of access and social involvement for elderly persons through physical activity.

Little information is available in Brazil about the involvement of the elderly in regular physical activities, whether targeted or spontaneous. A cursory examination of available studies also reveals that such information is localized in small areas of the country, and presents conflicting results regarding the possible prevalence of physical activities for the elderly. However, the few studies that exist put the physical inactivity levels of the Brazilian elderly population at an alarming $80-90 \%$.

In addition, it appears that information in literature regarding the effectiveness of national strategies to provide opportunities of spontaneous or supervised physical activities for the elderly is lacking. The data regarding the modalities of activities and the characteristics of physical activity programs in different regions of the country are, frankly, insufficient for the rational planning of what can be described as policies for the promotion of physical activity among this population. Studies focusing on the theme are therefore welcome and necessary.

In summary, there is much to be done in terms of public policies that allow, in the medium and long term, the involvement of Brazilian elderly persons in regular physical activity. Researchers who approach this theme can contribute to this task by producing studies that lead to a better understanding of the current status of the problem, and the development of strategies that are effective in terms of adhesion to physical activities and the impact on the functionality and quality of life of elderly persons with diverse clinical, social, economic and cultural characteristics.

Let's get to work! 